\title{
Web Accessibility Barriers in Geographic Maps
}

\author{
Tania Calle-Jimenez and Sergio Luján-Mora
}

\begin{abstract}
Geographic information (geo-information) is knowledge about where a place is or what there is in a certain site. Most people use geo-information in everyday life; for example, a map can be drawn to point out an address; a subway map can be interpreted to see which path to follow or to simply choose a route to go to work. Today, the Web is a mean of basic communication, perhaps the most important, and geographic information can also be transmitted through the Web. Therefore, we must ensure that the geographic information published on the Web is accessible. However, the continuing growth of technology causes people to have difficulty in interacting with applications that present geographic information. For this reason, this study presents an analysis of the barriers to web accessibility in geographic maps, explains how technologies and tools have evolved, and proposes the use of scalable vector graphics (SVG) for the implementation of accessible geographic maps.
\end{abstract}

Index Terms-Web accessibility, map, geographic tool, geographic information system.

\section{INTRODUCTION}

Accessibility to graphical information is basic even in initial education; since graphics are used for making elementary school children interpret its content. Graphical information is of fundamental importance in science, technology, engineering, mathematics, geography, history, social sciences, among others.

For example, graphical information is needed to interpret the content of geography, this information is represented by maps; school children learn about the world with maps, so you can say that the ability to read and examine maps is developed from the elementary school stage. Through the interpretation and study of different types of geographic maps, school children can understand more about economical, political, cultural, and social characteristics of the whole world.

Geographic maps are very important in everyday life and are present in multiple media, like television, Internet, newspapers and magazines. For example, for tourists who do not know where it is a specific site, they need to be guided and directed. That is, they need a map. Currently, we are witnessing a new era of geographic tools online: Google Maps, Google Earth, NASA World Wind, OpenStreetMap, MapQuest, Microsoft MapPoint are just a few examples. These tools have a large volume of users. For example, in the five largest European economies, $50 \%$ of Internet users

Manuscript received October 5, 2014; revised December 6, 2014.

Tania Calle-Jimenez is with National Polytechnic School, Faculty of Systems Engineering, Quito, Ecuador (e-mail: tania.calle@epn.edu.ec).

Sergio Luján-Mora is with University of Alicante, Department of Software and Computing Systems, Alicante, Spain (e-mail: sergio.lujan@ua.es). access online maps from their personal computers and 35\% of users do it on their mobile phones [1].

However, not all people can access geo-information on the Web. Due to the graphical nature of geo-information, some groups of users, such as low-vision or blind users; may experience problems when accessing geo-information.

According to a report that United Nations held in 2011, more than 1000 million people suffer some form of disability and the greater the poverty, the greater the exclusion [2]. In this work, we focus on people who have some kind of visual impairment. According to estimates by the World Health Organization (OMS), about 285 million people suffer from some form of visual impairment, which 39 million people are blind representing $0.7 \%$ of the world population [3].

Web accessibility means that people with disabilities will be able to make use of the web. Web accessibility is not interested in the specific conditions of the people but the impact these conditions have on their ability to use the Web [4].

This study presents an analysis of the barriers to web accessibility in geographic maps, how technologies and tools have evolved, and proposed to use Scalable Vector Graphics specification for design geographic maps accessible.

\section{BARRIERS TO WEB ACCESSIBILITY}

To achieve web accessibility, we need to be aware of the fact that not all users access the web with the same devices and also not all users are capable of correctly perceiving certain kinds of content published on the web. This general idea can be reflected in two basic design principles for web accessibility:

- To create pages that transform properly by offering content in different formats.

- To offer content in an understandable fashion to facilitate navigation through the website.

An example of these two principles is shown in the map displayed in Fig. 1. This map represents the subway system of London. This map contains "hot spots" that allow users to hear sound recorded in different places of the city. This map is accessible because of two reasons. On the one hand, there is an alternative text to replace audio features in the map. This map is accessible because a deaf person cannot hear the sound recording, but he can read the alternative text. On the other hand, the map can be accessed and activated by keyboard. Due to this, a blind person can use it as well.

Since several years ago, researchers have been working on web accessibility. As a result of this, some standards and guidelines have been defined to help developers to design accessible websites. One organization that has devoted more efforts to impulse web accessibility is the Web Accessibility Initiative (WAI), a working group that belongs to the Wide 
Web Consortium (W3C). WAI has defined different recommendations and guidelines related to web accessibility. Some of the best known guidelines are the Web Content Accessibility Guidelines 1.0 (WCAG 1.0), published in
1998, and its later version WCAG 2.0, published in 2008. The International Organization for Standardization (ISO) recognized WCAG 2.0 as international standard ISO/IEC 40500 in 2012.

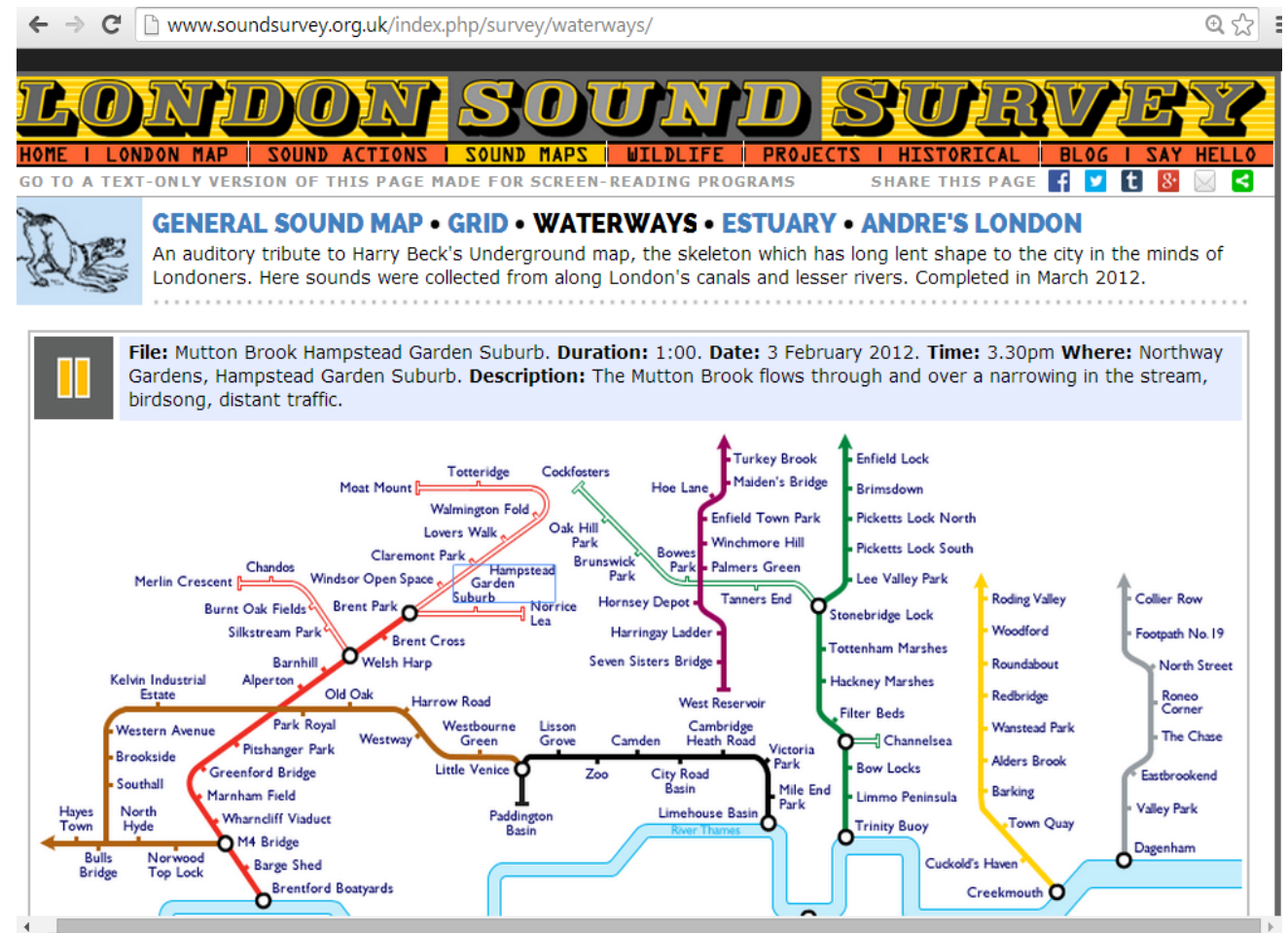

Fig. 1. Sound map accessible. ${ }^{1}$

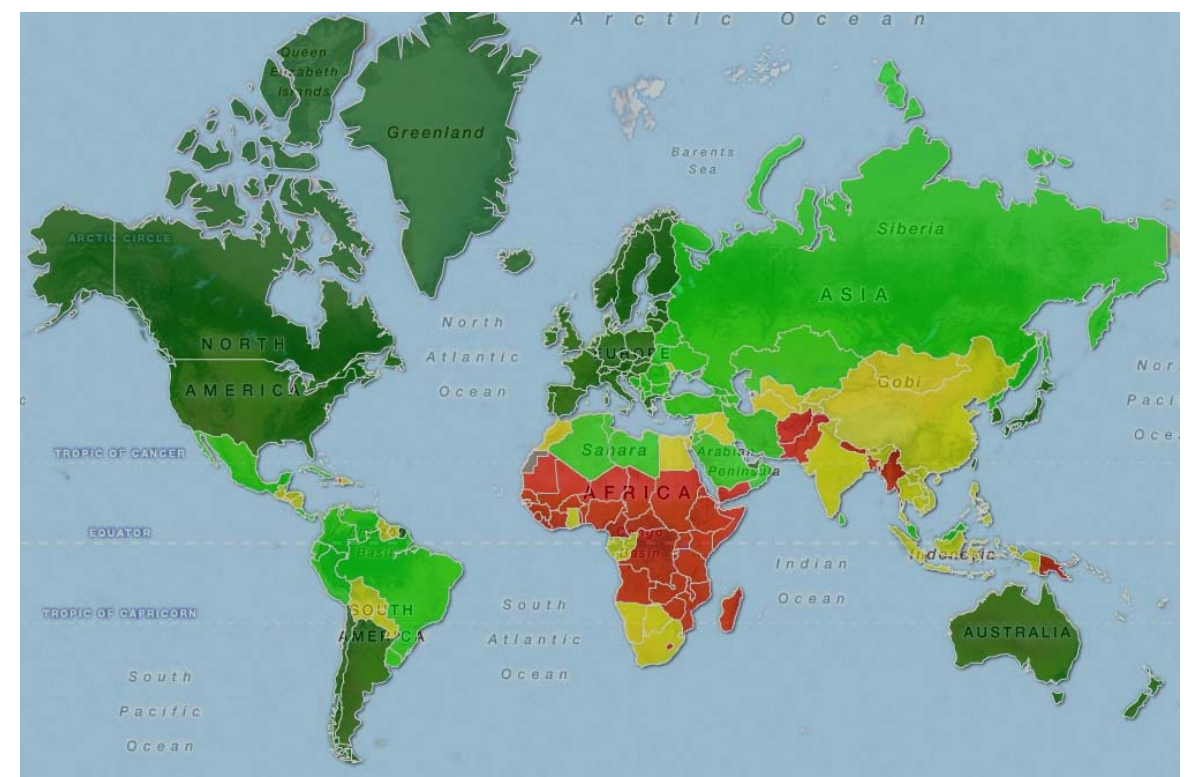

Fig. 2. Example of a thematic map that is not accessible.

In several countries, such as USA, UK, Spain and Japan, web accessibility standards have been legalized, because there is great awareness about the problems of people with disabilities and the goal is to defend their rights. Thanks to this, some improvements have been achieved, but there are still barriers that prevent implementing web accessibility in the geographic context. For example, the visualization of geo-referenced data is often offered by means of a hybrid display that combines abstract and concrete data. Issues related to the presentation of geo-referenced data are:
- Text represented as image: it means that the text that has an image is in image format.

- Not operable keyboard: it means that the functionality of the content of a website is not operable with keyboard.

- Problems with color: color blind people cannot distinguish certain shades of color.

- Mosaic maps: map consists of different images placed in an order so that they form a single image.

- Image with no text.

\footnotetext{
${ }^{1}$ http://www.soundsurvey.org.uk/index.php/survey/waterways/
} 
For example, the map shown in Fig. 2 represents the overall human development index (HDI). There is a scale of four types: dark green represents high values, light green shows a medium-high values, yellow represents medium-low values and red shows low values. This map is not accessible to a person with low vision or color blindness because the color differences used to transmit information are confusing both at visualizing and at printing. However, this map can be accessed if a better color scale and numbers are used to represent each type.

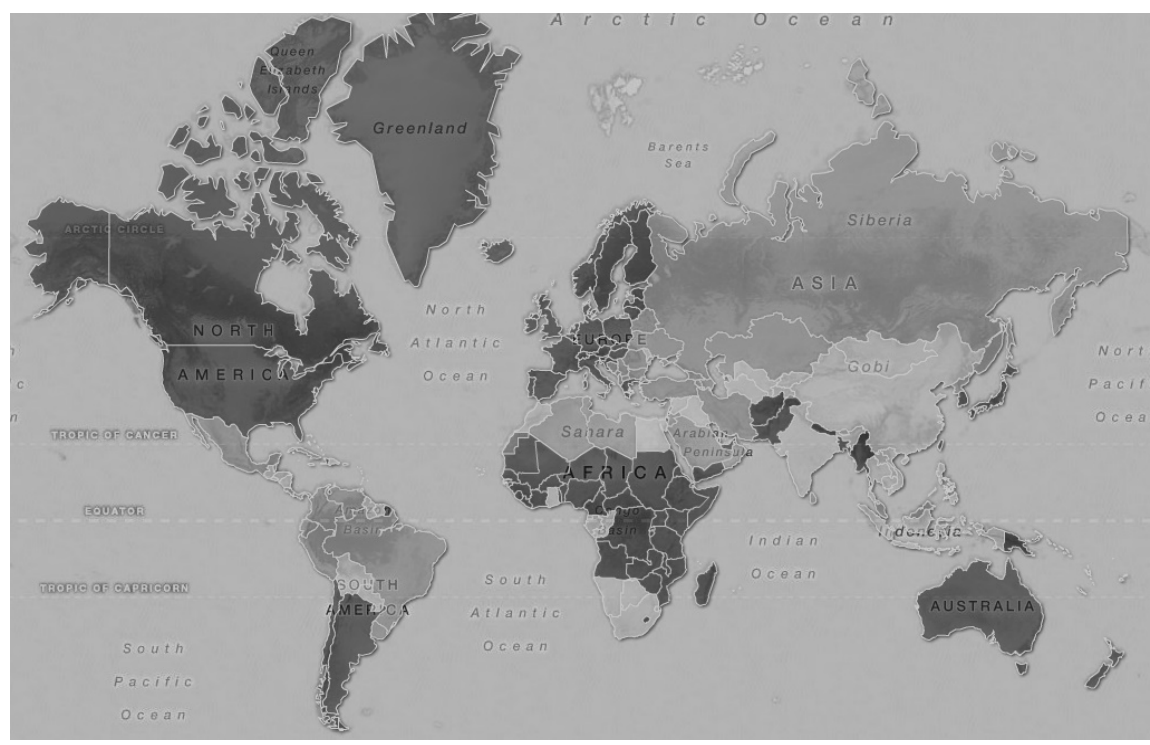

Fig. 3. Example of a thematic map that is not accessible.

If the map of Fig. 2 is printed in B/W, as shown in Fig. 3, it appears that the human development index of the United States is similar to Africa. This interpretation is inconsistent, and it can lead to misinterpretation. Hence, this map is not well designed for a person with low vision or blindness, and it is not accessible as shown in the map symbology in Fig. 4. However, this map could be accessible, if it would have a better color scale and use of numbers for each type.

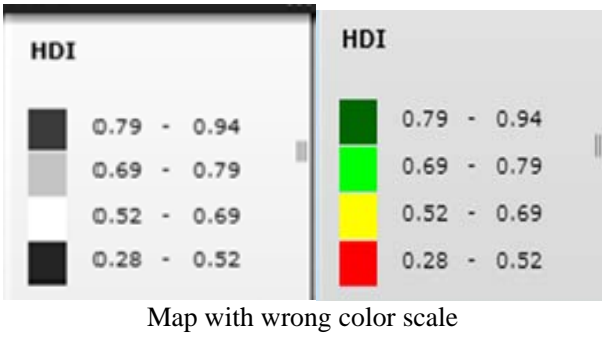

Fig. 4. Comparison between color scales.

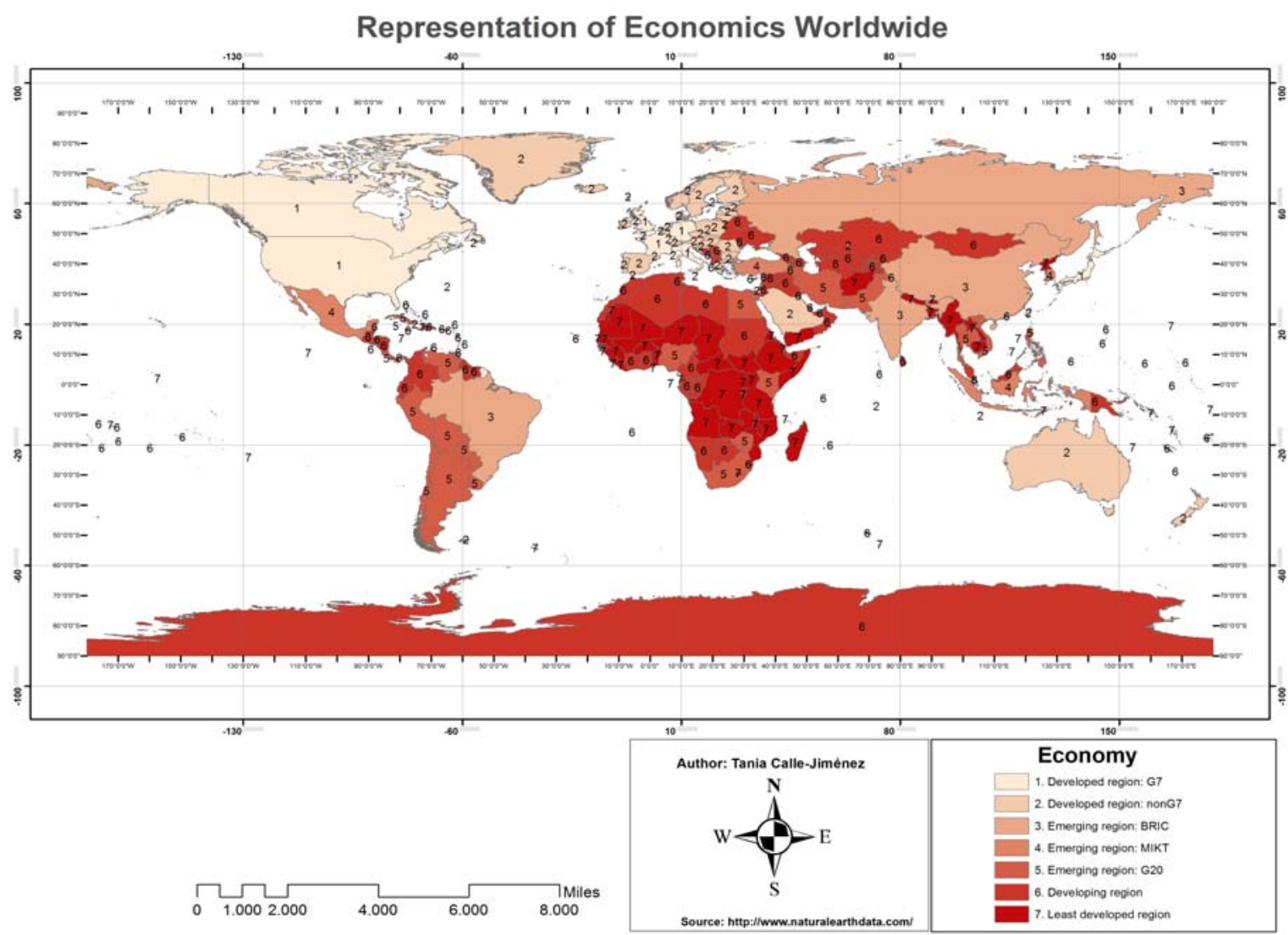

Fig. 5. Example of a thematic map with color that is accessible. 


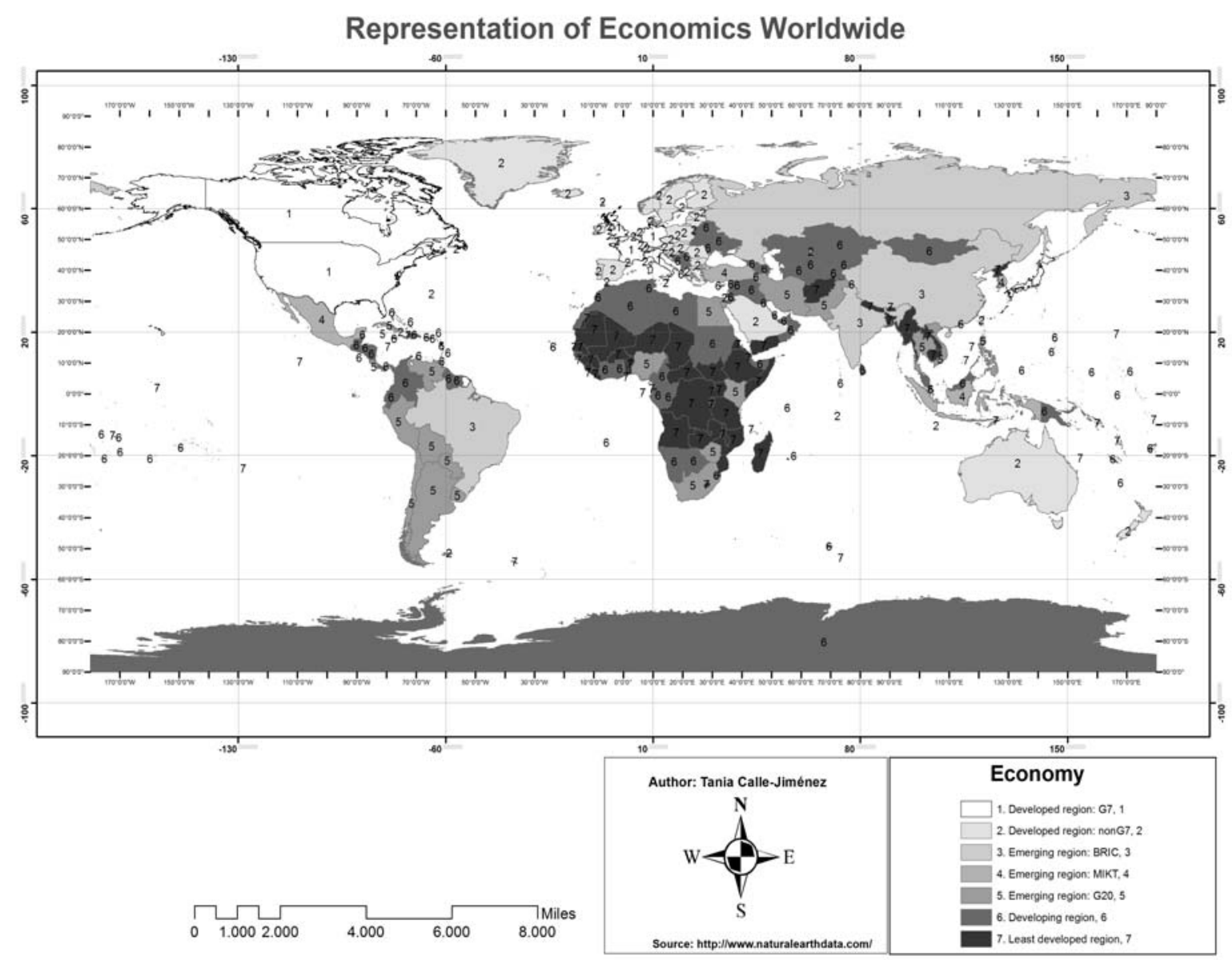

Fig. 6. Example of a thematic map with grayscale that is accessible.

Web accessibility is not only beneficial to people with disabilities, but also benefits all kind of users. For example, the map of Fig. 5 presents global economic information that displays the symbology with a ramp of red color, and additionally this map displays numbers to improve understanding.

If the map of Fig. 5 is printed in B/W, you have the map of Fig. 6, which shows a grayscale. In this case, this map is accessible for a person with low vision or blindness.

In Fig. 7 the symbology of the two previous examples shows a clear difference in types for both accessible maps.

\section{Economy}

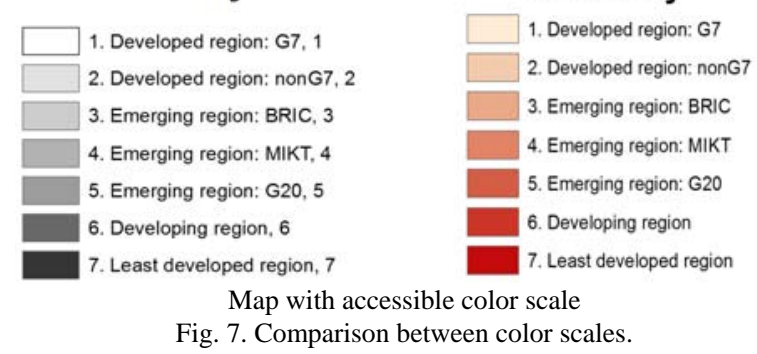

Most geographic information contained in websites is usually available only through images, which in general are not accessible for people with low vision. When information is only displayed through images and alternative text, the information become inaccessible to people with low vision. Different map formats shown on websites are:

- Maps only as image.

- Maps as a set of layers represented by lines, points and

\section{polygons.}

- Interactive maps.

- Maps with continuous information that changes over time.

Maps that are displayed in the web and have the features mentioned above, are not fully accessible. There are accessibility guidelines that deal with text, tabular, keyboard operated, color, etc. But there are some web accessibility problems of maps that are still not solved, such as how to represent geographic maps formed by layers, interactive maps, and maps with continuous information.

\section{EVOLUTION OF ACCESSIBILITY OF WEB MAPS}

Maps by their nature are inherently inaccessible to blind users because information on maps is transmitted primarily in a visual way. People with low vision use assistive technologies, such as text-to-speech screen readers which unfortunately cannot directly interpret geographic content.

There are several touch projects that allow low vision users navigate maps. There are also software that allow users to interact with maps using only the keyboard. Its main function is to summarize audio corresponding to the text that is displayed to the user, but this is not sufficient, since there are maps with lots of detail that cannot be interpreted with a voice system. Besides, providing a lot of audio information can produce a cognitive overload.

The attempt to convert geographic information to be accessible to all users and find a satisfactory alternative that 
allows browsing to people with vison problems, is a very active research field. There are several practical solutions developed by different authors, some of them are still prototypes, other are already implemented. There is a growing interest in applying the concept of web accessibility in maps. In the following sections, relevant works are described.

\section{A. Improving Accessibility of Web GIS Applications}

Vector graphics can be used to design geographic maps [Luaces M.R. et al., 2005]. This research presents a web service that enables the transformation of vector format from scalable vector graphics (SVG) to DHTML (HTML+JavaScript) in a new active map representation, as a mechanism to improve accessibility of web GIS applications. This representation includes a raster and a vector of a geographic map. The raster is used as the background image of the map, while the vector can respond to user events. An RTree structure is used to efficiently access the geographic objects affected by each event in order to execute the relevant action [5].

\section{B. Multimodal Eyes-Free Exploration of Maps}

TIKISI (Touch It, Key It, and Speak It) is a framework designed for users with low vision as an accessible mechanism for navigation of graphical information [Bahram, Sina et al., 2013]. In this paper, TIKISI has a multimodal input that includes keystrokes, spoken commands, and multi-touch gestures. TIKISI consists of three conceptual layers of mapping functions. The first set of mappings are not domain specific and abstracts out the various modalities of touch, keyboard, or speech input. The second set of mappings are domain specific and includes performing the required action, updating any internal state representations, and returning any results for presentation to the user. The third set of mappings then act upon these results (if results are produced), translating them into an appropriate form for the user. This third set of mappings is not strictly domain specific, but it is informed by the domain specific second layer.

The individual components of TIKISI are decoupled from one another to support flexibility in distributing responsibilities to different software components as well as extensibility (e.g., the possibility of multiple device and device interaction). With TIKISI, users with low vision can run a finger over a geographic map and issue commands to center, rescale, or zoom the map; go to specific locations, find features, etc. [6].

\section{Mobile GIS Based on SVG}

Mobile GIS applications are becoming very popular. The devices used to execute these applications suffer serious constrains in three areas: screen size, memory and speed. In this research [Wu Binzhuo et al., 2005] is presented a mobile GIS application based on Mobile SVG (Scalable Vector Graphics) for hand held devices with limited resources.

The study case is a mobile application of tourism. This application adopts mobile SVG as carrier to data, display and parser of map. Mobile SVG profiles introduce constraints on content, such as attribute types, properties, and user agent behavior, due to low memory, low power and limited display.
SVG parses files and gets data of map then displays the SVG file as a map. The paper describes the application's data format, presents the map and points out the areas for future development [7].

Mobile GIS can help people with disabilities to move around cities and other places, both outdoors and indoors. For example, blind people can use a mobile GIS to find the directions to arrive at a chosen place. Or a person with motor impairment (e.g. a person in a wheelchair) can use a mobile GIS to find an accessible route in an airport or railway station [8].

\section{Audio-Tactile Exploration of Interactive Maps}

This article investigates whether vibration and speech feedback can be used to improve accessibility of digital maps on touch devices. The authors tested the vibration feedback combined with movement of the user's finger over relevant map objects [Poppinga B. et al., 2011].The results indicate that it is possible to get a basic overview of the map layout without having access to the visual presentation [9].

\section{E. Touching OpenStreetMap Data in Mobile for Low Vision Users}

In this paper, authors present an application that enables access to OpenStreetMap data for blind and low vision users using mobile devices [Kaklanis N. et al., 2013]. During the exploration, the user moves his finger on the touchscreen of the mobile device and receives vibration feedback when finger is on a road or a point of interest. Sonification and a text-to-speech module provide audio feedback about distance to the next crossroad and information of the road or point of interest [10].

\section{F. Final Reflection}

As noted in the previous works, currently there is growing interest in web accessibility for maps. For example, in 2003 ESRI - owner of the ArcGIS platform- showed its interest in web accessibility saying that it is good for the GIS industry and proposed strategies for developing accessible GIS. Two main strategies were: developing products, technologies and services that are accessible and usable by all people; and empowering customers with information to make informed decisions about accessibility capabilities in ESRI products. Also, the ArcGis version 9.x have incorporated the exportation of maps in the SVG format [11].

\section{TeChnOlOgY And TOOLS}

Fortunately, in last years the interest to improve web accessibility has increased. Some of the new existing technologies that allow presenting both graphics and text on websites are:

- Hypertext Markup Language version 5 (HTML5). It specifies two variants of HTML text and XHTML syntax. It is still in experimental mode, although it is already used by many web developers for its advances, improvements and advantages, including SVG, JavaScript, and XML to present dynamic data in the web.

- Cascading Style Sheets (CSS). It is a language used to describe the look and formatting of a document written in 
a markup language, including several XML-based languages such as XHTML or SVG.

- Web Accessibility Initiative - Accessible Rich Internet Applications (WAI-ARIA). It is a technical specification published by the World Wide Web Consortium that specifies how to improve the accessibility of web pages, in particular, dynamic content and user interface components developed with Ajax, HTML, JavaScript and related technologies [12].

- Scalable Vector Graphics (SVG). It is a specification for describing two-dimensional vector graphics, both static and animated. SVG allows three types of graphic objects such as vector geometric elements represented by points, lines and polygons, digital bitmap images, and text.

With the advancement of tools and programming technologies, it has appeared new ways of representing dynamic graphical content as shown by JavaScript, HTML5, SVG, and CSS. Not only is important to present dynamic content on the web, but also is essential to make this content accessible. WAI-ARIA presents an overview of how to make web pages accessible, especially in the dynamic part, guiding the developers to implement accessible websites.

\section{Proposal of Using SVG to Design ACCESSIBle GEOGRAPHIC MAPS}

SVG aims to solve the problem of loss of definition that an image suffers when it is resized, while maintaining the size of the file as compact as possible. Using SVG has recently become very popular in developing websites that contain many images.

SVG includes some elements which provide supplementary descriptive information. A SVG map may consist of several components combined hierarchically, each subcomponent is included as a container element $\langle$ g $>$ with an id attribute and a text equivalent, specified with $<$ title $>$ and $<$ desc $>$ elements. These elements can be children of any graphic or container element in SVG. The $<$ title $>$ is meant for a short text description of an element. If the text description is complex, the <desc $>$ element should be used because it is intended to provide arbitrarily long descriptions (nothing in the SVG specification limits the length of these elements). These elements can be read out with screen readers. <desc> and $<$ title $>$ elements are not rendered as part of the SVG graphics. However, the <title> element can be visually displayed when the pointing device moves over or selects particular elements. As an example, a code segment of an SVG map is displayed in Fig. 8. This map is composed of some polygons and lines that together form a map. This map is a schematic representation of buildings, parking lots, streets and green areas (Fig. 9).

The whole map has supplementary information provided by <title> "Ecuador University" and < desc> "Escuela Politécnica Nacional University-Ecuador” elements. Besides, each part of the map includes a container element, such as <g id="EEParkingLot" $>$, that has its supplementary title ("Electronic Engineering Parking Lot") and description ("Parking for teachers of Electrical Engineering"). Inside this element, there is another container that described the elements $<\mathrm{g}$ id="EEParkingLotPlaces" $>$ that contain $<$ title $>$
"Place 1", and description <desc> "Parking place for Electrical Engineering professor, area $2.5 \times 4$ meters”.

The title and the description can be used to improve the accessibility of the map: a screen reader can retrieve the textual information of the map, which can be provided to people with visual disabilities. The container element $<\mathrm{g}>$ can be used to organize the content of the map in different levels (layers) that can be offered to the user on demand.

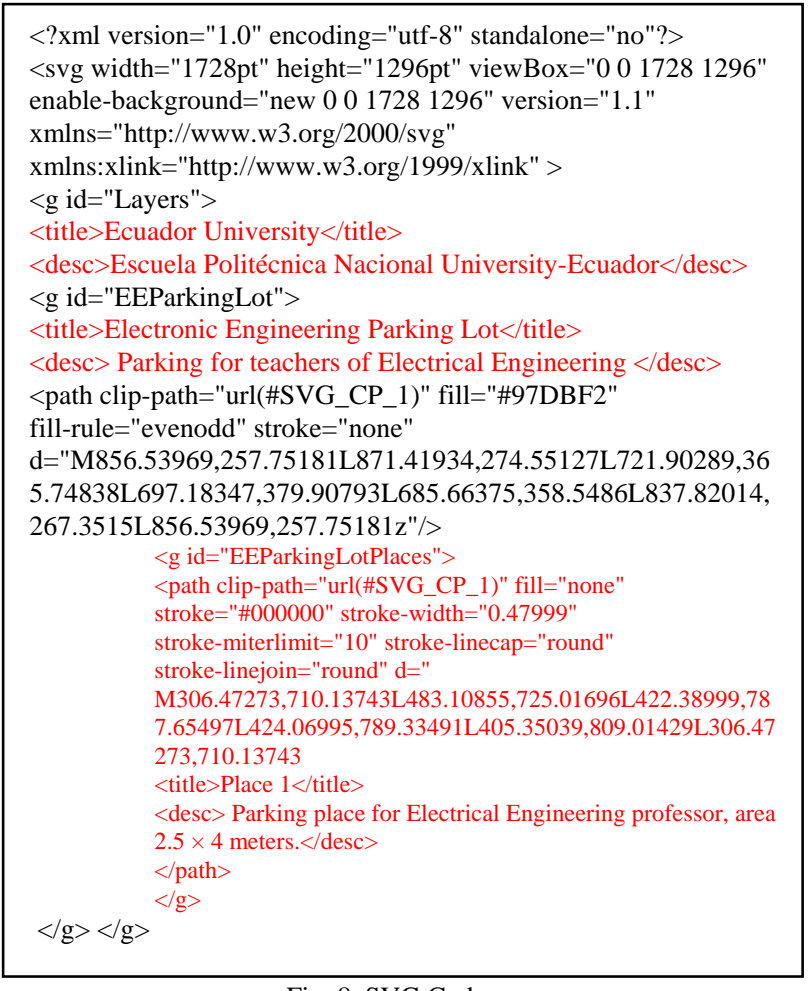

Fig. 8. SVG Code.

\section{FUTURE WORK}

There is still a lot to do to improve the accessibility of maps in the geographic web, especially regarding platforms and design of maps.

Platforms include: base hardware, software frameworks, virtual machines, cloud environments, virtualizations, and browsers, among others. The main challenges regarding platforms are:

- To develop services and technologies accessible and usable by all people.

- To apply web accessibility guidelines to implement operating systems, web servers, DBMS, map servers.

- To implement appropriate architectures containing a web accessibility service.

What remains to be done regarding design of maps, i.e. with respect to the map visualization is:

- To establish accessibility levels to differentiate among a first stage of visual maps, a second stage of legible maps, and a final stage of fully accessible maps. Guidelines should be extended to include this graduation in levels of accessibility.

- To focus on the specificity of the map function and the user of the map. For example, design a bump map is not the same as making a street map, since the two types of 
maps need different criteria and resources.

- To develop methods to provide more accessible information in the form, scale, and level of detail required by the user.

- To meet the needs of users requiring multi-scale representations.

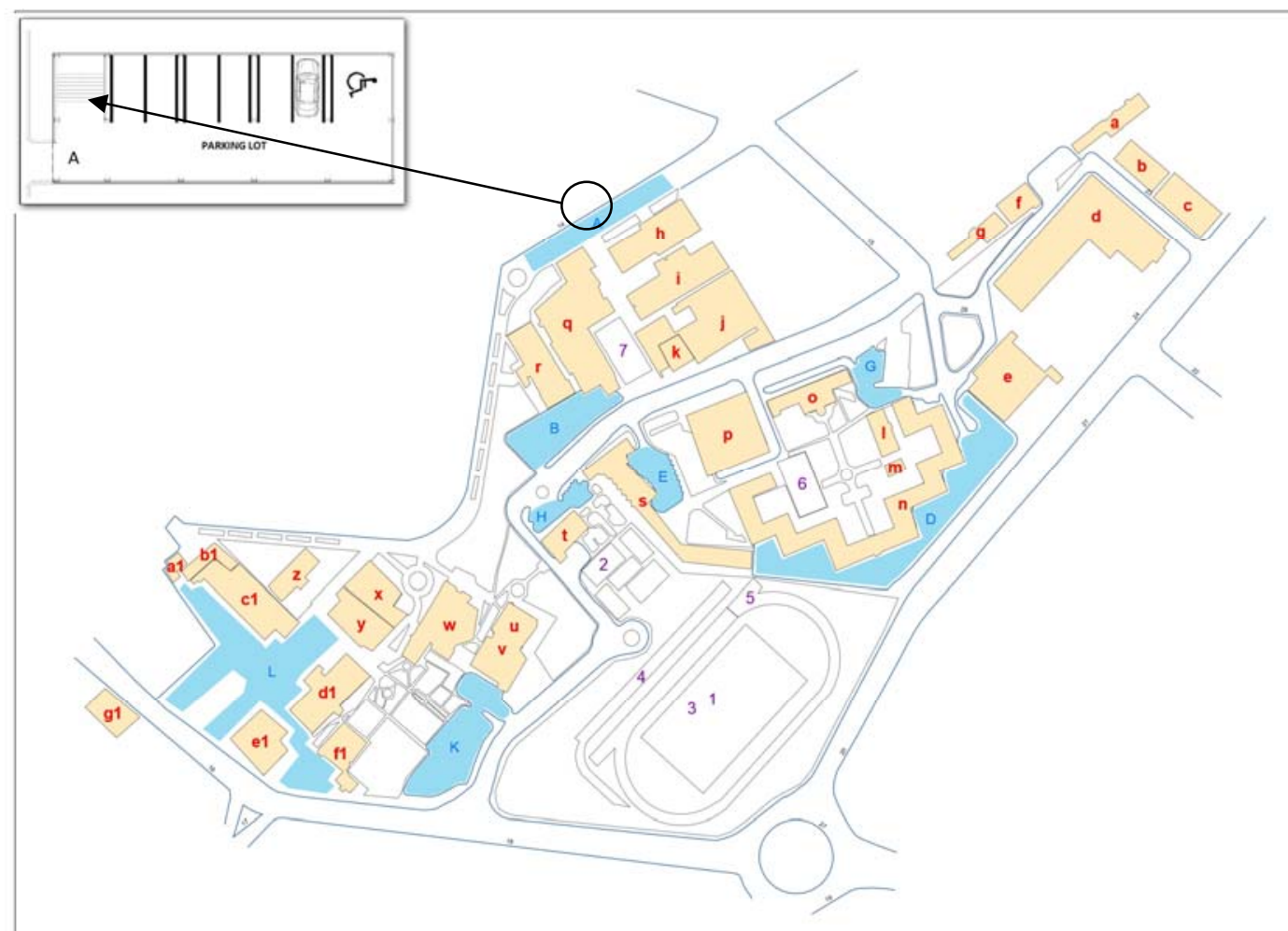

Fig. 9. Result of the SVG Code.

\section{CONCLUSIONS}

New online geographic applications, such as OpenStreetMap and Google Maps make clear the need for using the geographic information in a very different way to the commonly uses of printed maps. However, it is necessary to develop methods that take in account form, scale and detail to provide more accessible information.

Most studies reviewed in the literature, have little or no interest in applying web accessibility guidelines in the development of geographic platforms, since the maps are usually not accessible and strictly visual. This shows a current weakness in geographic web development.

This study presented some elements of the SVG format as $<$ g $>$, <desc $>$ and <title $>$ labels, for describing geographic map elements such as polygons, lines and points. This allows that SVG code can be interpreted by a screen reader that can read each map form using the title and description; so users with visual disabilities can interpreter and manipulate the geographic map.

Even though that SVG format was created to represent images with dynamic content, SVG also provides its own characteristics in the code to develop accessible geographic maps as shown in this study. As future works, we intend to combine SVG format with Web Accessibility Guidelines and another vector formats to obtain new ways to access and display the maps on the Web.

Development of accessible geographic systems using the guidelines WCAG 2.0 can help people with disabilities to be able to locate places on a map without the support of another people.

\section{ACKNOWLEDGMENT}

This work has been partially supported by the Prometeo Project by SENESCYT, Ecuadorian Government.

We would like to appreciate the invaluable help of Sandra Sanchez-Gordon in the review of this work.

\section{REFERENCES}

[1] Google, What Is the Economic Impact of Geo Services, Oxera, 2013.

[2] World Health Organization. (2013). Word report on disability. [Online]. Available:

http://www.who.int/disabilities/world_report/2011/en/index.html

[3] International Agency for the Prevention of Blindness (IAPB). (2010). Technical report. [Online]. Available: http://www.iapb.org/sites/iapb.org/files/State\%20of\%20the\%20World \%20Sight_2010.pdf

[4] World Wide Web Consortium (W3C). (2013). Introduction to web accessibility. [Online]. Available: http://www.w3.org/WAI/intro/accessibility.php

[5] N. R. Brisaboa, "Improving accessibility of web-based GIS applications," in Proc. Sixteenth International Workshop on Database and Expert Systems Applications, Aug. 2005, pp. 490-494.

[6] S. Bahram, "Multimodal eyes-free exploration of maps: TIKISI for maps,” ACM SIGACCESS Accessibility and Computing Homepage archive, issue 106, pp. 3-11, June 2013.

[7] B. Wu and X. Bin, "Mobile phone GIS based on mobile SVG," in Proc. Geoscience and Remote Sensing Symposium, July 2005, vol. 8, pp. 5527-5529.

[8] B. Poppinga, "Touchover Map: audio-tactile exploration of interactive maps,” MobileHCI, Stockholm, Sweden, Aug. 30-Sept. 2, 2011.

[9] N. Kaklanis et al., "Touching open street map data in mobile context for the visually impaired," in Proc. CHI 2013 Mobile Accessibility Workshop, Paris France, April 28, 2013.

[10] D. Leithinger and H. Ishii, "Relief: A scalable actuated shape display," TEI, Cambridge, Massachusetts, USA, January 25-27, 2010.

[11] ESRI. (2003). Delivering accessible internet GIS services. [Online]. Available: http://www.esri.com/news/arcuser/0206/denver2of2.html 
[12] World Wide Web Consortium (W3C). (2014). Accessible Rich Internet Applications (WAI-ARIA) 1.0. [Online]. Available: http://www.w3.org/TR/wai-aria/

[13] World Wide Web Consortium (W3C). (2014). Accessibility Features of SVG. [Online]. Available: http://www.w3.org/TR/SVG-access

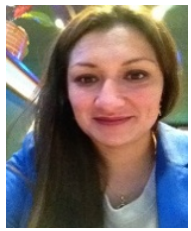

Tania E. Calle Jiménez was born in Quito, Ecuador on December 31, 1980. She earned her degree in systems engineering in 2006; she has a master degree in geographic information systems in 2011. She also has a master degree in geomatics in 2012.

Currently, she is a professor at the National Polytechnic School and a PhD student at University of Alicante, Spain

Her Main relevant publications are: "Web accessibility evaluation of massive open online courses on geographic information systems" in Proc.
IEEE Global Engineering Education Conference, EDUCON 2014 and "Retos de Accesibilidad en GEO-MOOCs," in Proc. Ibero-American Conference WWW/Internet CIAWI 2013.

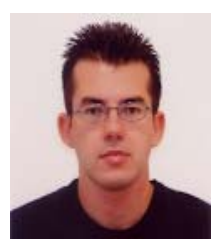

Sergio Luján Mora obtained his $\mathrm{PhD}$ degree in computer engineering from the University of Alicante with a research on modeling of data warehouses using UML. Currently, he is a professor in the Department of Computer Languages and Systems, University of Alicante, and a researcher on the area of web accessibility. 